\title{
IMPACT OF EXPERIMENTAL NEONATAL PROPOFOL ANAESTHESIA ON NEURODEVELOPMENTAL SHORT- AND LONG-TERM OUTCOME
}

\author{
G.W. Schlager ${ }^{1}$, T. Karen ${ }^{1}$, I. Bendix ${ }^{1}$, M. Sifringer ${ }^{2}$, R. Herrmann ${ }^{1}$, C. Pantazis $^{1}$, S. Prager $^{1}$, M. Keller ${ }^{1}$, U. \\ Felderhoff-Müser ${ }^{1}$ \\ ${ }^{I}$ Dept. of Paediatrics I, University Hospital Essen, Essen, ${ }^{2}$ Dept. of Anaesthesia and Intensive Care, Charité \\ Universitätsmedizin Berlin, Berlin, Germany
}

Propofol is commonly used as sedative in neonatology. As recent experimental studies led to contradictory results, showing neurodegenerative or neuroprotective short-term properties of Propofol on the developing brain, we aimed to investigate the neurodevelopmental short- and long-term effects of neonatal Propofol treatment.

7 day old Wistar rats (P7) randomised in two groups received either three i.p. injections $(0,90,180 \mathrm{~min})$ of saline (1) or $30 \mathrm{mg} / \mathrm{kg}$ Propofol (2). Animals were sacrificed 6, 12 and $24 \mathrm{hr}$ after the first administration. Cortical and thalamic areas were dissected and analysed by Western-Blot and RT-qPCR. Long-term effects were assessed by a series of behavioural tests (Open-field, Novel-Object-Recognition) at P30 and P120.

Western-Blot analyses of cortical areas showed a transient increase of activated Caspase-3, as well as a reduction of active mitogen-activated protein kinases ERK1/2 and AKT in cortical and thalamic areas. RTqPCR analyses determined a downregulation of neurotrophic factors BDNF and NGF in cortical and thalamic regions. Assessment of the behavioural phenotype showed no impairment in memory formation or anxiety but revealed hyperactive behaviour in Propofol treated adolescent animals (P30).

Our results point to a transient increase of apoptosis following Propofol administration in our animal model. Assessment of long-term neurodevelopment revealed hyperactivity in Propofol treated animals on P30. Current investigations will determine whether this is still present in adult animals (P120). In combination with previous studies, our preliminary findings suggest that Propofol should be used with caution in the neonatal period, and that the neurodevelopmental outcome should not be underestimated in clinical trials. 